\title{
Simulation in vitro des fermentations cæcales du lapin en fermenteur à flux semi-continu. I. Rôle du prétraitement du substrat alimentaire
}

\author{
D Adjiri 1, M Bouillier-Oudot 1, F Lebas 2, M Candau 1 \\ ${ }^{1}$ Ecole nationale supérieure agronomique de Toulouse, laboratoire de zootechnie \\ et de productions animales, 145, avenue du Muret, 31076 Toulouse Cedex; \\ 2 INRA, laboratoire de recherches sur l'élevage du lapin, BP 27, \\ 31326 Castanet-Tolosan Cedex, France
}

(Reçu le 8 janvier 1992; accepté le 30 juin 1992)

\begin{abstract}
Résumé - Un fermenteur à flux semi-continu de type Rusitec a été utilisé pour étudier l'influence de prétraitements enzymatiques du substrat alimentaire sur le profil fermentaire observé au cours des 2 semaines suivant l'inoculation avec du contenu cæcal de lapin. Trois types de substrat ont été introduits dans le fermenteur : un aliment commercial pour lapins, simplement broyé; la partie solide de cet aliment, restant après un traitement de $24 \mathrm{~h}$ par une $\alpha$-amylase; le substrat 2 ayant subi en plus un traitement de $4 \mathrm{~h}$ par de la pepsine (double traitement enzymatique). Chaque jour, l'un des 2 sachets nylon contenant $15 \mathrm{~g}$ du substrat étudié est remplacé par un nouveau, ce qui conduit à une durée de fermentation uniforme de $48 \mathrm{~h}$. En 5-6 j, la fermentation de l'aliment non traité conduit à un profil fermentaire totalement différent de celui observé in vivo dans un cæcum de lapin : I'acide propionique dépasse $35 \%$ des acides gras volatils (AGV) totaux et l'acide butyrique représente environ $15 \%$ de ces derniers. Le traitement par l'amylase seule conduit à un profil fermentaire stable plus proche de celui observé in vivo, mais où les acides propionique et butyrique ont des proportions très voisines, proches de $15 \%$ des AGV totaux. Le double traitement par l'amylase et la pepsine induit un faciès fermentaire stable, tout à fait comparable à celui observé in vivo: $\mathrm{C}_{2}>60 \% ; \mathrm{C}_{3}<11 \%$ et $17 \%<\mathrm{C}_{4}<21 \%$. Pour ce dernier substrat, le taux de disparition des constituants membranaires (ADF de Van Soest) en $48 \mathrm{~h}$, est comparable au coefficient de digestibilité mesuré in vivo par d'autres auteurs pour le même aliment de base. Enfin, il convient de signaler la présence d'un taux élevé d'acides gras volatils à 5 carbones (environ $5 \%$ ) qui pourrait être due au mode discontinu d'approvisionnement du fermenteur : un sachet nouveau y est en effet introduit toutes les $24 \mathrm{~h}$. En conclusion, un double prétraitement de l'aliment par une $\alpha$-amylase et de la pepsine permet de simuler in vitro les fermentations cæcales du lapin dans un fermenteur semi-continu de type Rusitec.
\end{abstract}

\section{lapin / cæcum artificiel / substrat / prétraitement / acide gras volatil}

Summary - In vitro simulation of rabbit caecal fermentation in a semi-continuous flow fermentor. 1. Effect of food substrate pretreatment. A Rusitec semi-continuous flow fermentor was used to study the influence of enzyme pretreatment of food substrates on the fermentation profile over a 2-week period following inoculation with rabbit caecal contents. Three types of substrate were examined: 1) homogenized commercial rabbit feed; 2) the solid remains of this feed after digestion with $\alpha$-amylase for $24 h$; and 3) substrate 2 digested for $4 h$ with pepsin (double enzyme treatment). One of a pair of nylon pouches containing $15 \mathrm{~g}$ substrate was replaced each day, thus producing a uniform 48-h fermentation. Fermentation of the untreated feed (1) for 5-6 days produced a fermenta- 
tion profile quite different from that obtained in vivo in the rabbit caecum: propionic acid accounted for over $35 \%$ of total volatile fatty acid (VFA), and butyric acid for about $15 \%$. Amylase digestion (2) gave a stable ferment profile closer to the in vivo profile, except that propionic and butyric acids were similar at $15 \%$ of total VFA. Digestion with both amylase and pepsin (3) produced a stable fermentation profile very close to the in vivo profile: $C_{2}>60 \%, C_{3}<11 \%$ and $17 \%<C_{4}<21 \%$. The rate at which membrane constituents (acid detergent fibre, ADF) were lost in $48 \mathrm{~h}$ was similar to the digestibility coefficient measured in vivo by others for the same basic feed. Lastly, there was a high percentage (about 5\%) of volatile $C_{5}$ fatty acids; this could be due to the discontinuous fermentor input of one pouch per $24 \mathrm{~h}$. Thus, feed pretreated with both amylase and pepsin simulates, in vitro, rabbit caecal fermentation in a semi-continuous Rusitec type fermentor.

\section{rabbit / artificial caecum / substrate / initial treatment / volatile fatty acid}

\section{INTRODUCTION}

Chez le lapin, les processus digestifs sont dominés par la succession d'une dégradation enzymatique dans l'intestin grêle avec absorption des produits issus de cette dégradation, suivie d'une fermentation du bol alimentaire dans le cæcum. Contrairement aux autres herbivores monogastriques (cheval, cobaye) et aux ruminants en général, le profil des acides gras volatils (AGV) du contenu du fermenteur digestif est caractérisé, chez le lapin, par une proportion d'acide butyrique supérieure à celle de l'acide propionique.

Les troubles digestifs du lapin sont presque systématiquement associés à une perturbation de cet équilibre des AGV (Prohaszka, 1980; Morisse et al, 1985). Il est donc nécessaire de bien en analyser le déterminisme. L'étude in vivo des paramètres susceptibles d'influencer la teneur et la proportion des AGV est difficile, en particulier, en raison des problèmes de canulation cæcale et surtout des artefacts associés aux prélèvements trop rapprochés. L'observation in vivo est même parfois impossible pour certains métabolites rapidement absorbés à travers la paroi cæcale. Par contre, l'étude in vitro des facteurs de variation du profil fermentaire peut être un outil d'investigation intéressant dans la mesure où une perturbation de la flore n'entraîne pas de réaction de la part de «l'hôte».
Des travaux en fermenteur clos ont été publiés par différents auteurs (Tafani, 1981; Salse, 1985). Ils permettent d'étudier par exemple la vitesse initiale d'hydrolyse, mais rapidement les fermentations sont inhibées par l'accumulation des métabolites issus de l'activité de la flore elle-même. Ce modèle de fermenteur in vitro ne peut donc permettre d'étudier que les paramètres pouvant influencer l'activité de la flore dans les quelques heures suivant la fermeture du système. Par voie de conséquence, ils ne sont pas adaptés aux études sur une longue période.

C'est pourquoi nous nous proposons de mettre au point un cæcum artificiel sous forme d'un fermenteur «ouvert» permettant des études sur plusieurs jours et pouvant ainsi devenir l'outil d'investigation mentionné plus haut. Pour accélérer la mise au point de ce cæcum artificiel, nous sommes partis d'un fermenteur semi-continu du type Rusitec (Czerkawski et Brekenridge, 1977). Rappelons simplement que dans ce type de fermenteur, le matériel alimentaire solide est introduit périodiquement et qu'un flux continu de "salive artificielle» permet une évacuation continue des produits de la fermentation.

Le présent travail vise à déterminer les conditions de préparation du substrat alimentaire introduit chaque jour dans le fermenteur, de manière telle que le profil fermentaire des AGV soit stable et conforme à celui du lapin : prédominance de l'acide acétique et teneur en acide butyrique supé- 
rieure à celle de l'acide propionique. À cette fin, et pour estimer le rôle de la digestion dans l'intestin grêle sur les caractéristiques du profil fermentaire, nous avons comparé l'effet d'un traitement de l'aliment par de l'amylase, suivi ou non par un traitement par de la pepsine, en comparaison avec le profil observé in vitro avec l'aliment brut non traité.

\section{MATÉRIEL ET MÉTHODES}

\section{Nature et réalisation des substrats}

Trois types de substrat ont été réalisés. Le premier est un aliment de type commercial "lapincroissance" (substrat ALIMENT) constitué de $40 \%$ de luzerne deshydratée, $30 \%$ d'orge, $10 \%$ d'avoine, $10 \%$ de tourteau de tournesol, $5 \%$ de tourteau de soja et $5 \%$ d'un complément minéral vitaminisé. II est employé après un simple broyage des granulés (grille de $1 \mathrm{~mm}$ ).

Le $2^{e}$ substrat est obtenu à partir de cet aliment par un prétraitement à l'amylase (substrat AMYL). Ce dernier est réalisé de la manière suivante : un mélange de $750 \mathrm{~g}$ d'aliment broyé et de $60 \mathrm{~g}$ d'amylase (EC 3.2.1.1. Sigma réf A3176) dans 3,75 I d'eau est mis à incuber sous agitation à $39^{\circ} \mathrm{C}$ durant $24 \mathrm{~h}$. Ce mélange est ensuite filtré sur une toile à bluter de $150 \mu \mathrm{m}$ de vide de maille. Le résidu est lavé, puis séchè à $60^{\circ} \mathrm{C}$.
Le $3^{\theta}$ substrat correspond au même aliment soumis à l'hydrolyse amylasique précédente, suivie d'une dégradation par la pepsine (substrat AMYL-PEPS). Après l'incubation en présence d'amylase et avant filtration, le produit est l'objet d'une acidification jusqu'à pH 1,5 par action d'acide chlorhydrique. II est ensuite incubé $4 \mathrm{~h}$ dans les mêmes conditions, en présence de $15 \mathrm{~g}$ de pepsine (EC 3.4.23.1 Merk réf 7190). Le $\mathrm{pH}$ du mélange est ensuité élevé entre 5 et 6 par de la soude, avant filtration, lavage et séchage du résidu.

\section{Appareillage de fermentation}

Comme indiqué plus haut, le système de fermentation utilisé est celui mis au point par Czerkawski et Brekenridge en 1977 pour simuler les fermentations dans le rumen. L'équipement comprend 4 fermenteurs indépendants, en altuglass, de 1 I de volume et maintenus dans un bain thermostaté à $39^{\circ} \mathrm{C}$. Les substrats solides sont contenus dans 2 sachets de $15 \times 8 \mathrm{~cm}$, fabriqués avec un tissu de nylon ayant un vide de maille de $150 \mu \mathrm{m}$. Ces sachets sont introduits dans un flacon plastique perforé et soumis à un mouvement linéaire alternatif à l'intérieur du fermenteur. Une solution saline (tableau I) est apportée en continu et les effluents liquides et gazeux évacués par trop plein. Seuls les permiers sont récupérés.

Devant le peu dinformation disponible concernant la composition minérale des contenus ileaaux et cæcaux du lapin, la composition de la so-

Tableau I. Composition de la solution saline infusée dans les fermenteurs.

\begin{tabular}{|c|c|}
\hline $\begin{array}{l}\text { Bicarbonate de sodium }\left(\mathrm{NaHCO}_{3}\right) \mathrm{mg} / \mathrm{l} \\
\text { Phosphate disodique }\left(\mathrm{Na}_{2} \mathrm{HPO}_{4}, 12 \mathrm{H}_{2} \mathrm{O}\right) \mathrm{mg} / \mathrm{l} \\
\text { Chlorure de sodium }(\mathrm{NaCl}) \mathrm{mg}^{\prime l} \\
\text { Chlorure de potassium }(\mathrm{KCl}) \mathrm{mg} / \mathrm{l} \\
\text { Chlorure de calcium }\left(\mathrm{CaCl}_{2} \text { anhydre }\right) \mathrm{mg} / \mathrm{l} \\
\text { Chlorure de magnésium }\left(\mathrm{MgCl}_{2}, 6 \mathrm{H}_{2} \mathrm{O}\right) \mathrm{mg} / \mathrm{l} \\
\text { Sulfate de sodium }\left(\mathrm{Na}_{2} \mathrm{SO}_{4}\right) \mathrm{mg} / \mathrm{l} \\
\text { Sulfate de zinc }\left(\mathrm{ZnSO}, 7 \mathrm{H}_{2} \mathrm{O}\right) \mathrm{mg} / \mathrm{l} \\
\text { Chlorure de cobalt }\left(\mathrm{CoCl}_{2}, 6 \mathrm{H}_{2} \mathrm{O}\right) \mathrm{mg} / \mathrm{l} \\
\text { Sulfate de manganèse }\left(\mathrm{MnSO}_{4}, \mathrm{H}_{2} \mathrm{O}\right) \mathrm{mg} / \mathrm{l} \\
\left.\text { Sulfate ferreux FeSO } \mathrm{Fi}_{4}, \mathrm{H}_{2} \mathrm{O}\right) \mathrm{mg} / \mathrm{l} \\
\text { Sulfate de cuivre }\left(\mathrm{CuSO}_{4}, 5 \mathrm{H}_{2} \mathrm{O}\right) \mathrm{mg} / \mathrm{l}\end{array}$ & $\begin{array}{l}9240 \\
7125 \\
470 \\
450 \\
55 \\
72 \\
22,20^{\mathrm{a}} \\
4,40 \\
1,20 \\
19 \\
36,80 \\
0,98\end{array}$ \\
\hline
\end{tabular}

\footnotetext{
a La quantité de sulfate de sodium varie en fonction de la quantité d'azote uréique apportée : $47,3 \mathrm{mg} / \mathrm{h}$ pour $\mathrm{AMYL}$,
} 58,5 pour AMYL-PEPS 
lution saline dérive de celle utilisée pour la simulation des fermentations du rumen en Rusitec (Blanchart et al, 1989). Seule la teneur en phosphate a été modifiée pour obtenir un $\mathrm{pH}$ voisin de celui du contenu iléal du lapin $(\mathrm{pH}>7,5-8)$. Cette solution doit permettre d'assurer le pouvoir tampon du milieu ainsi que les apports indispensables en oligoélements pour assurer la croissance microbienne.

\section{Ensemencement et mise en œuvre des fermentations}

Chaque fermenteur est ensemencé à partir du contenu de 5 cæca provenant de lapins nourris avec un aliment comparable à l'aliment expérimental. Après sacrifice des animaux, les contenus cæcaux sont recueillis dans $300 \mathrm{ml}$ de mélange eau-solution saline $(2 / 1, v / v)$, maintenu à $39^{\circ} \mathrm{C}$ sous barbotage de $\mathrm{CO}_{2}$. Le mélange est filtré sur 4 couches de gaze et $400 \mathrm{ml}$ de filtrat sont introduits dans le fermenteur, constituant l'inoculum liquide. Le contenu du fermenteur est ensuite complété à $1 \mathrm{I}$ avec le mélange eau-solution saline. Environ $100 \mathrm{~g}$ de résidu solide de filtration contenus dans un sachet de nylon sont introduits dans le fermenteur pour constituer l'inoculum solide. Simultanément, est introduit le $2^{e}$ sachet avec $15 \mathrm{~g}$ de substrat expérimental. Après fermeture du fermenteur, une perfusion de solution saline assure, pendant toute la durée de l'observation, un renouvellement de la phase liquide de $0,5 \mathrm{l} / \mathrm{j}$.

Vingt quatre heures après la mise en route, le sachet contenant l'inoculum solide est remplacé par un sachet contenant $15 \mathrm{~g}$ de substrat. Chaque jour est ensuite assuré le renouvellement du sachet ayant séjourné $48 \mathrm{~h}$ dans le fermenteur.

Les 2 fermenteurs recevant les substrats AMYL et AMYL-PEPS reçoivent en outre, une complémentation azotée journalière, calculée de manière à assurer un apport global de $17 \mathrm{~g}$ de MAT pour $100 \mathrm{~g}$ de substrat, apport voisin de celui du contenu iléal du lapin. Cette complémentation n'est pas nécessaire pour l'aliment non traité. Elle est assurée pour moitié sous forme d'urée dans la solution minérale et pour moitié sous forme de peptone de caséine introduite directement dans le fermenteur.

\section{Paramètres étudiés}

Le $\mathrm{pH}$ est mesuré journellement dans chaque fermenteur au moment de l'ouverture.
Dans le volume d'effluent recueilli chaque $24 \mathrm{~h}$, sont déterminées les concentrations en azote ammoniacal par colorimétrie, en azote protéique (dosage de l'azote total sur le culot de centrifugation obtenu après précipitation à l'acide trichloracétique à $10 \%$ ) et en AGV par chromatographie en phase gazeuse (Jouany, 1982).

Sur les résidus des sachets, sont déterminées les teneurs en matière sèche, en matière organique et les constituants pariétaux NDF et ADF (Van Soest et Wine, 1967), de manière à estimer la dégradation de chaque constituant sur $48 \mathrm{~h}$ (DMS, DMO, DNDF, DADF).

\section{Analyse mathématique des résultats}

À partir des données recueillies chaque jour sur chaque fermenteur, les valeurs moyennes par fermenteur ont été comparées par analyse de variance selon un schéma factoriel incluant les effets fermenteur et jour de contrôle (bibliothèque statistique SAS-micro). Les moyennes sont fournies avec indication de l'écart type de la moyenne $\left(m \pm s_{m}\right)$.

\section{RESULTATS ET DISCUSSION}

La composition chimique des substrats utilisés dans les fermenteurs est présentée au tableau II en référence à la composition chimique du contenu de l'iléon de lapins recevant le même aliment de base (valeurs relevées par Gidenne et Ruckebusch en 1989). L'aliment non traité présente une teneur en amidon très supérieure à celle du contenu iléal, un niveau de matières azotées totales très voisin et une teneur en constituants pariétaux (NDF) inférieure. Après traitement enzymatique, la concentration en amidon est fortement réduite. Elle approche celle du contenu iléal. Par contre, la teneur en fibres des 2 substrats $A M Y L$ et $A M Y L-P E P S$ est supérieure à celle du contenu iléal. Après une phase d'adaptation de 4-5 j, l'utilisation des substrats AMYL et AMYL-PEPS a pour conséquence un $\mathrm{pH}$ du milieu fermentaire supé- 
Tableau II. Composition chimique des différents substrats. Comparaison avec la composition du contenu iléal déterminée par Gidenne et Ruckebusch (1989).

\begin{tabular}{lrrrr}
\hline Substrat & ALIMENT & AMYL & AMYL-PEPS & "léon \\
& & & & \\
g/kg MS & & & & \\
Matière organique & 906 & 930 & 912 & $834(6)$ \\
MAT & 175 & 92 & 74 & $155(6)$ \\
Amidon & 207 & 15 & 14 & $13(2)$ \\
NDF & 336 & 665 & 691 & $455(20)$ \\
ADF & 205 & 435 & 474 & $307(20)$ \\
\hline
\end{tabular}

a Valeurs moyennes et erreur standard entre parenthèses pour prélèvements iléaux sur 6 lapins alimentés avec l'aliment non traité (ALIMENT) (Gidenne et Ruckebusch, 1989)

rieur à celui obtenu avec l'aliment non traité $(6,5-6,8$ vs 6,4$)$, et supérieur au $\mathrm{pH}$ obsenvé in vivo $(5,8-6,2)$.

\section{Production azotée}

L'évolution journalière de l'azote protéique recueilli dans les effluents suit, quel que soit le substrat utilisé, une courbe de type exponentiel décroissant (fig 1). Après une chute continue durant les 5 ou 6 premiers

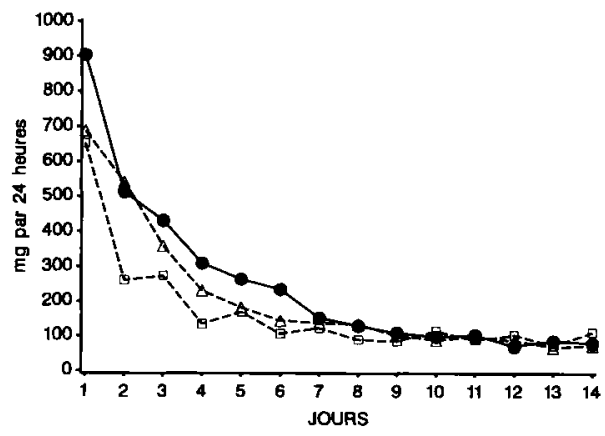

Fig 1. Influence du prétraitement enzymatique du substrat sur l'évolution journalière de l'azote protéique $(\mathrm{mg} / \mathrm{j})$ recueilli dans l'effluent du cæcum artificiel. Fermenteur ALIMENT : cercles pleins; fermenteur AMYL : carrés vides; fermenteur AMYL-PEPS : triangles vides. jours, on observe une stabilisation au voisinage de $100 \mathrm{mg} / \mathrm{j}$ quel que soit le fermenteur considéré (tableau III).

Malgré une possible contamination des effluents par de l'azote issu du substrat (Blanchart et al, 1989), l'azote protéique de l'effluent peut être assimilé pour l'essentiel à de l'azote microbien (Fuller et Johnson, 1981). On observe donc, dans les premiers jours de fermentation, un lessivage microbien. Celui-ci semble lié au fait qu'à un inoculum important $(50 \mathrm{~g}$ de MS environ) et riche en matériel microbien issu des contenus cæcaux de 5 lapins, seulement $15 \mathrm{~g}$ de maitère sèche sont apportés journellement par fermenteur, soit un flux nettement inférieur à celui observé in vivo au niveau cæcal : de l'ordre de $80-100 \mathrm{~g}$ de MS entrent chaque jour dans le cæcum, pour un contenu représentant 25-30 g de MS. Cette chute de la population microbienne, induite par une diminution drastique de la disponibilité en substrat, se poursuit jusqu'à obtention de l'équilibre entre flux alimentaire et croissance microbienne. Celui-ci semble survenir au bout de $6 \mathrm{j}$ environ.

Toutefois, l'estimation de l'azote microbien de l'effluent (approché par l'azote protéique) ne constitue qu'une fraction de l'azote microbien produit. Le complément 
Tableau III. Influence du traitement enzymatique du substrat sur les paramètres de dégradation et fermentaires en cæcum artificiel*.

\begin{tabular}{|c|c|c|c|}
\hline & ALIMENT & $A M Y L$ & AMYL-PEPS \\
\hline $\mathrm{pH}$ & $6,40 \pm 0,04^{b}$ & $6,68 \pm 0,03^{a}$ & $6,67 \pm 0,04^{a}$ \\
\hline $\mathrm{N}-\mathrm{NH}_{3} \mathrm{mg} / \mathrm{j}$ & $35,2 \pm 10,4^{c}$ & $181,0 \pm 16,8^{b}$ & $220,0 \pm 19,2^{a}$ \\
\hline N-Prot mg/i & $102,9 \pm 26,7$ & $98,0 \pm 17,4$ & $97,5 \pm 25,6$ \\
\hline AGV mmol/ & $95,2 \pm 6,2^{\mathrm{a}}$ & $80,7 \pm 7,3^{b}$ & $83,0 \pm 3,3^{b}$ \\
\hline $\mathrm{C}_{2} \%$ & $42,7 \pm 1,7^{b}$ & $62,5 \pm 1,1^{\mathrm{a}}$ & $64,1 \pm 1,1^{a}$ \\
\hline $\mathrm{C}_{3} \%$ & $35,9 \pm 3,2^{a}$ & $14,2 \pm 0,9^{b}$ & $10,3 \pm 1,2^{c}$ \\
\hline $\mathrm{IsoC}_{4} \%$ & $1,0 \pm 0,1^{b}$ & $1,0 \pm 0,1^{b}$ & $1,2 \pm 0,0^{a}$ \\
\hline $\mathrm{C}_{4} \%$ & $15,4 \pm 3,9^{b}$ & $16,3 \pm 0,5^{a}$ & $18,8 \pm 1, \gamma^{a}$ \\
\hline $1 \mathrm{soC}_{5} \%$ & $1,0 \pm 0,2^{b}$ & $2,8 \pm 0,2^{a}$ & $2,8 \pm 0,1^{a}$ \\
\hline $\mathrm{C}_{5} \%$ & $4,0 \pm 0,5^{\mathrm{a}}$ & $3,1 \pm 0,1^{b}$ & $2,8 \pm 0,2^{b}$ \\
\hline
\end{tabular}

* Valeurs moyennes obtenues sur 8 j de fermentation, après 6 j d'adaptation. a, b, c Les moyennes associées à des lettres différentes sont significativement différentes au seuil $P<0,001$, sur une mème ligne.

est représenté par les microrganismes attachés au substrat solide resté dans les sachets nylon. La détermination de cette fraction supposerait la séparation du matériel microbien du substrat (technique du "stomacher») et/ou le dosage des substances microbiennes spécifiques (ARN, acide diaminopimélique), ou encore l'emploi de marqueur $\left({ }^{15} \mathrm{~N}\right)$. Ceci n'ayant pas été envisagé dans cette première étape, nous ne pouvons pas établir de bilan de la synthèse microbienne dans nos fermenteurs.

Contrairement à l'azote protéique dont la production est indépendante du substrat mis à fermenter, les quantités d'azote ammoniacal recueillies dans les effluents des fermenteurs alimentés avec les substrats AMYL et AMYL-PEPS sont, après la période d'adaptation, très supérieures à celles recueillies pour l'aliment non traité (181 et 220 vs $35 \mathrm{mg} / \mathrm{j}$ ) (tableau III, fig 2). Cette production plus élevée d'N- $\mathrm{NH}_{3}$ peut être attribuée essentiellement à la complémentation importante en urée apportée avec les substrats prétraités. Celle-ci est rapidement hydrolysée par les microrganismes cæcaux entraînant un excès relatif d'ammoniaque non utilisé pour les synthèses microbiennes. Cet écart de production peut être relié au $\mathrm{pH}$ plus faible constaté avec le substrat ALIMENT. Par ailleurs, on doit remarquer que la concentration moyenne en $\mathrm{N}-\mathrm{NH}_{3}$ dans les fermenteurs AMYL et AMYL-PEPS, - 360$440 \mathrm{mg} / \mathrm{l}$-, est comparable à celle obser-

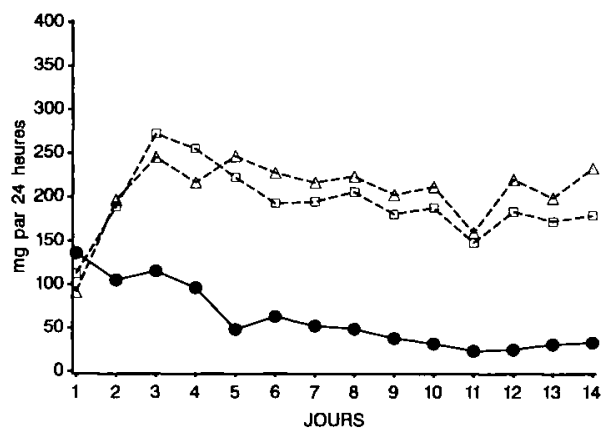

Fig 2. Évolution quotidienne de l'azote ammoniacal recueilli dans l'effluent des 3 fermenteurs $(\mathrm{mg} / \mathrm{j})$. Fermenteur ALIMENT : cercles pleins; fermenteur AMYL : carrés vides; fermenteur AMYL-PEPS : triangles vides. 
vée in vivo dans le contenu cæcal, - 360$560 \mathrm{mg} / \mathrm{l}$-, par Gidenne (1986). En conséquence, c'est la teneur en $\mathrm{N}-\mathrm{NH}_{3}$ associée au substrat ALIMENT qui doit être considérée comme réduite in vitro par rapport à la valeur in vivo. II est alors logique de penser à une plus faible activité bactérienne associée à ce substrat, du moins pour les bactéries productrices d'N-NH $\mathrm{N}_{3}$, malgré un flux azoté total comparable. La qualité de ce flux azoté, essentiellement protéique, est probablement en cause.

\section{Production d'AGV}

En présence du substrat ALIMENT, les premiers jours de fermentation font apparaitre, pour les acides gras volatils, un profil assimilable à celui aes fermentations cæcales. II y a bien prédominance de l'acide butyrique $\left(\mathrm{C}_{4}\right)$ sur l'acide propionique $\left(\mathrm{C}_{3}\right)$ avec toutefois une proportion faible d'acide acétique $(50 \%)$ en regard de celle observée in vivo. Cependant ce profil n'est pas stable dans le temps (fig 3a), et dès le $6^{e}$ jour de fermentation, le rapport butyrate/propionate s'inverse en faveur de l'acide propionique qui atteint une valeur élevée $\left(C_{3}>35 \%\right)$. Parallèlement, la teneur en $\mathrm{C}_{4}$ diminue lentement. Après une dizaine de jours, on observe un faciès fermentaire similaire à celui du rumen d'un polygastrique alimenté avec un régime riche en amidon.

En présence de l'aliment traité uniquement avec l'amylase (fig 3b), les proportions molaires des différents AGV apparaissent beaucoup plus stables au cours du temps. La proportion d'acide acétique $\left(C_{2}\right)$ est supérieure à $60 \%$, valeur comparable à celle observée in vivo dans le cæcum du lapin $(60-80 \%$ en fonction des conditions d'observation). Effectivement, Lang (1981) et Morisse et al (1985) mentionnent des proportions de $\mathrm{C}_{2}$ évoluant de

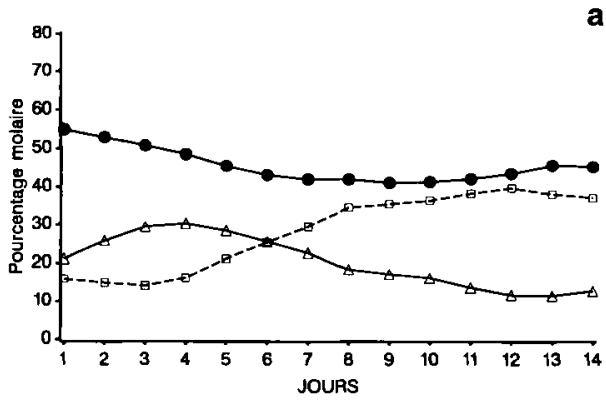

b
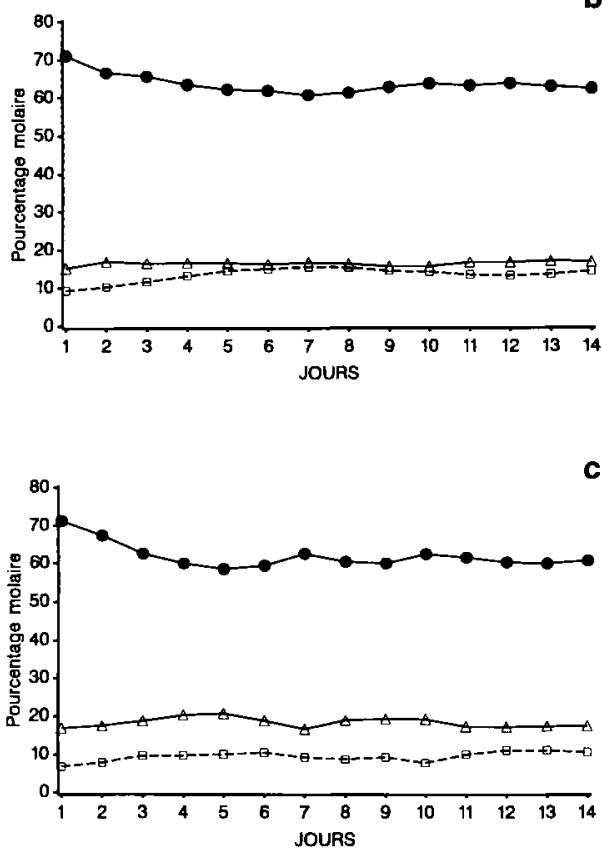

Fig 3. Répartition des principaux acides gras volatils produits journellement dans chacun des 3 fermenteurs : a) substrat ALIMENT, b) substrat AMYL, c) substrat AMYL-PEPS. Acide acétique : cercles pleins; acide propionique : triangles; acide butyrique : carrés.

60 à $65 \%$, tandis que Fioramonti et Ruckebusch (1976) et Gidenne et al (1991) observent des proportions d'acide acétique allant jusqu'à $78 \%$. Par contre, malgré une 
prédominance légère mais significative $(P<0,001)$ de l'acide butyrique, les niveaux de celui-ci et de l'acide propionique sont très voisins et se stabilisent autour de $15 \%$. Cette valeur est relativement élevée pour de lacide propionique provenant de fermentations cæcales.

En présence de l'aliment traité par l'amylase et la pepsine, les fermentations conduisent à un faciès d'AGV (fig 3c) typique des fermentations cæcales du lapin, avec une grande stabilité sur l'ensemble de la période de fermentation $\left(\mathrm{C}_{2}>60 \%\right.$, $\mathrm{C}_{3}<11 \%$ et $17 \%<\mathrm{C}_{4}<21 \%$ ).

Par ailleurs, quel que soit le substrat utilisé, les fermentations se caractérisent par un niveau élevé des acides $\mathrm{C}_{5}$ et iso $\mathrm{C}_{5}$ $(1,0-2,8 \%$ et $2,8-4 \%$, respectivement). Nous avons également détecté la présence de $\mathrm{C}_{6}$, mais les teneurs n'ont pas été quantifiées. Selon Kolb (1975), l'acide valérique ainsi observé pourrait provenir du catabolisme des protéines, et en particulier de la lyse bactérienne. Bien que peu d'auteurs mentionnent les teneurs en ces acides gras volatils chez le lapin, nos observations sont en contradiction avec celles de Dehalle et Lebas (1981) et Gidenne (1986) qui, pour les lapins alimentés à volonté, mentionnent des teneurs inférieures à $1 \%$ en $\mathrm{C}_{5}$ et iso $\mathrm{C}_{5}$. Cependant, Demaux et al (1981) observent, en fin de repas chez les animaux nourris en un repas par jour, une élévation au-delà de $8 \%$ des AGV totaux, pour l'ensemble $C_{5}$, iso $\mathrm{C}_{5}$ et $\mathrm{C}_{6}$. Cette proportion redescend à $3 \%$ après $6 \mathrm{~h}$. Au vu de nos résultats, l'utilisation dans notre cas d'une technique de fermentation en semi-continu s'apparente semble-t-il à cette dernière conduite alimentaire.

Enfin, la concentration moyenne des acides gras volatils totaux (tableau III) apparaît légèrement, mais significativement plus élevée en présence de l'aliment non traité. Ceci est à relier à la présence d'ami- don et de sucres solubles rapidement fermentés, alors que ceux-ci ont été en majeure partie éliminés par les traitements subis par les deux autres substrats plus riches en fibres.

\section{Conditions générales de fermentation}

Nos différents résultats ont été obtenus chaque fois avec 2 semaines d'observations quotidiennes, mais avec un seul fermenteur par type de substrat. Toutefois, les travaux conduits en parallèle par l'un d'entre nous (Adjiri, données non publiées) ont déterminé la répétabilité entre fermenteurs des profils fermentaires. Pour un même substrat et des conditions identiques, les écarts observés entre fermenteurs pour les différents paramètres fermentaires en général et les proportions d'AGV en particulier sont toujours nettement inférieures à celles observés ici en fonction du substrat, y compris la différence entre AMYL et AMYL-PEPS pour les proportions de $\mathrm{C}_{3}$ et de $\mathrm{C}_{4}$. Nos observations sont donc généralisables.

Ainsi, seul le prétraitement par l' $\alpha$ amylase et la pepsine, simulant la digestion enzymatique pré-cæcale, nous a permis de maintenir in vitro des fermentations dont le faciès se rapproche de celui des fermentations cæcales du lapin. La présence d'amidon semble inhiber la production d'AGV. Compte tenu du taux de disparition des constituants membranaires du substrats AMYL-PEPS (DNDF de 24,5 \pm $5,6 \%$ et DADF de $17,4 \pm 6,8 \%$ ) nous pouvons penser que les $A G V$ observés dans l'effluent ont pour origine principale la dégradation microbienne des constituants fibreux. Nous devons également souligner que notre coefficient moyen de disparition de ADF est tout a fait comparable à celui observé in vivo pour le même aliment : CUDa de 15,0 $\pm 2,6 \%$ (Gidenne et Ruckebusch, 1989). 
La seule élimination de la majeure partie de la fraction amylacée n'est pas suffisante pour assurer un substrat adéquat simulant le contenu iléal entrant dans le cæcum. L'élimination d'une fraction de l'azote protéique alimentaire insoluble dans l'eau et/ou la modification structurelle de la fraction restante, à la suite du traitement par la pepsine, s'avère aussi utile pour orienter les fermentations vers un faciès cæacal. Les aspects quantitatifs de l'apport azoté ne semblent pas en cause, dans la mesure où l'azote alimentaire éliminé par le prétraitement pepsique est remplacé par une quantité équivalente d'azote sous forme uréique et peptidique. Entre les 2 substrats AMYL et AMYLPEPS, les complémentations azotées ainsi réalisées sont assez proches : 7,8 $\mathrm{g}$ pour $100 \mathrm{~g}$ du premier substrat et $9,6 \mathrm{~g}$ pour $100 \mathrm{~g}$ du second. Aussi, il nous semble peu probable que ce modeste écart d'apport uréique et de peptone puisse entraîner à lui seul la différence sensible de faciès observée pour les acides butyrique et propionique. Considérer cette modification du faciès comme la conséquence de l'effet du traitement pepsique sur la modification de la structure protéique nous paraît plus vraisemblable, mais nous ne pouvons, dans le cadre de cette expérience, en apporter la preuve formelle.

\section{CONCLUSION}

En utilisant un fermenteur semi-continu, à partir d'un inoculum constitué par du contenu cæcal et en employant un substrat préalablement traité par voie enzymatique, nous avons réussi à maintenir sur l'ensemble d'une période d'observation de 2 semaines, des fermentations présentant, sur la base des profils en AGV produits, les principales caractéristiques fermentaires cæcales du lapin.
À partir d'un aliment de type commercial, un double traitement enzymatique par l'amylase et la pepsine visant à simuler les dégradations pré-cæcales apparaît nécessaire pour fournir un substrat adéquat se rapprochant du contenu iléal et assurer ainsi un profil de fermentation de type cæcal.

Si l'élimination de la majeure partie de l'amidon alimentaire ne semble pas suffisante pour obtenir un substrat adéquat, le traitement par la pepsine apparaît indispensable, plus par les transformations qualitatives qu'il entraîne que par la diminution de l'azote du substrat.

Enfin, si nous avons mis en évidence la possibilité de simuler in vitro les fermentations cæcales du lapin, ce travail ne constitue qu'une première étape de mise au point qu'il est nécessaire de poursuivre pour mieux cerner les conditions de mise en œuvre et ainsi s'approcher au plus près des conditions physiologiques du cæcum in vivo. De nombreux points restent encore obscurs. II faudra par exemple connaître le rôle précis de l'origine de l'inoculum employé sur le faciès fermentaire : serait-il le même avec un inoculum ruminal appliqué au substrat AMYL-PEPS, qu'avec un inoculum cæcal de lapin ? Sa masse initiale devra également faire l'objet d'investigations. II conviendra aussi de bien déterminer le rôle des apports azotés complémentaires utilisés, en quantité et en nature. Enfin une adaptation technique devrait être trouvée pour permettre d'étudier des temps de séjour en fermenteur beaucoup plus courts et proches des conditions physiologiques (8-12 h). Sous cet angle, en dehors des contraintes d'emploi du temps, la principale difficulté actuelle est la rupture relative d'anaérobiose associée à chaque ouverture de la cuve de fermentation pour y introduire le ou les nouveaux sachets.

En conclusion, cette technique semble être un outil perfectible mais déjà efficace, 
devant contribuer à l'avenir à l'étude du rôle des substrats entrant dans le cæcum, sur l'activité fermentaire qui s'y développe.

\section{RÉFÉRENCES}

Blanchart G, Durand $M$, Barry JL, BouillierOudot $M$, Jouany JP (1989) Intérêts et limites des fermenteurs à flux semi-continu de type Rusitec dans l'étude des fermentations du rumen. Ann Zootech 38, 285-314

Czerkawski JW, Brekenridge G (1977) Design and development of a long-term rumen simulation technic (Rusitec). Br J Nutr 38, 371-384

Dehalle C, Lebas $F(1981$ ) Incidence de régimes alimentaires à base de luzeme sur la composition en acides gras volatils du contenu digestif chez le lapin. Reprod Nutr Dev 21, 867

Demaux G, Guémon L, Gallouin F (1981) Temporal changes of some biochemical and histological parmeters at different levels in the rabbit digestive tract. Reprod Nutr Dev 21, 868

Fioramonti J, Ruckebusch Y (1976) La motricité cæcale chez le lapin. III. Dualité de l'excrétion fécale. Ann Rech Vét 7, 281-295

Fuller JR, Johnson DE (1981) Monensin and lasalocid effects on fermentations in vitro. J Anim Sci 53, 1574-1580

Gidenne T, Ruckebusch Y (1989) Flow and passage rate studies at the ileal level in the rabbit. Reprod Nutr Dév 29, 403-412

Gidenne T (1986) Évolution nycthémérale des produits de la fermentation bactérienne dans le tube digestif du lapin en croissance. Relations avec la teneur en lignine de la ration. Ann Zootech 35, 121-136

Gidenne T, Scalabrini F, Marchais C (1991) Adaptation digestive du lapin à la teneur en constituants pariétaux du régime. Ann Zootech 40, 73-84

Jouany JP (1982) Dosage des acides gras volatils (AGV) et des alcools dans les contenus digestifs, le jus d'ensilage, les cultures bactériennes et les contenus de fermenteurs anaérobies. Sci Alim 2, 131-144

Kolb E (1975) Physiologie des animaux domestiques. Éditions Vigot Frères, Paris

Lang $\mathrm{J}$ (1981) The nutrition of commercial rabbit. I. Physiology, digestibility and nutrient requirements. Nutr Abstr Rev 51, 197-225

Morisse JP, Boilletot E, Maurice R (1985) Alimentation et modifications du milieu intestinal chez le lapin (AGV, $\mathrm{NH}_{3}, \mathrm{pH}$, flore). Rec Méd Vét 161, 443-449

Prohaszka $L$ (1980) Antimicrobial effect of volatile fatty acids in enteric Escherichia coli infections of rabbits. Zbl Vet Med B27, 631-639

Salse A (1985) Development of an artificial cecum and quality of the obtained product. Reprod Nutr Dev 32, 491-501

Tafani JP (1981) Étude de l'utilisation digestive des fibres alimentaires chez le lapin par la technique du cæcum artificiel. Thèse de Doctorat vétérinaire, Fac Méd Créteil, $111 \mathrm{p}$

Van Soest PJ, Wine RH (1967) Use of detergents in the analysis of fibrous feeds. IV. Determination of plant cell wall constituents. $J$ Assoc Offic Agric Chem 50, 50-55 